\title{
Adsorção do Violeta de Cristal em Areia
} Uma experiência simples para ilustrar os conceitos de adsorção.

\section{Enquadramento}

A importância do ensino experimental em Química é amplamente reconhecida. No entanto em algumas áreas escasseiam exemplos de trabalhos simples, seguros e económicos, realizáveis em períodos curtos (3-4h).

A adsorção é um dos tópicos usualmente leccionados em disciplinas de Química-Física nas licenciaturas de Química, Engenharia Química e Engenharia do Ambiente. Ao nível do ensino secundário o tema é abordado como base para o ensino da cromatografia não sendo objecto de qualquer estudo experimental. Todavia a adsorção é um tema actual com elevado potencial de aplicação para a redução do impacto ambiental de várias indústrias. Como exemplo cite-se a remoção de cor de efluentes corados tais como os efluentes das indústrias têxteis, da indústria papeleira e de algumas indústrias alimentares.

0 trabalho aqui apresentado pode servir como exemplo do tratamento de um efluente corado e, simultaneamente, permite ilustrar os princípios básicos de adsorção.

\section{Fundamentos teóricos}

O fenómeno de adsorção consiste na transferência de um ou mais solutos de um fluido para a superfície de um sólido, o adsorvente. Esta interacção soluto/sólido deriva da existência de forças não compensadas à superfície do sóli- do, resultando numa distribuição de equilíbrio do soluto entre as duas fases.

Consoante a natureza das forças envolvidas pode falar-se em adsorção quimica ou adsorção física. Num processo de adsorção química há formação de ligações químicas entre o soluto e o sólido, formando-se uma única camada de moléculas adsorvidas - monocamada. 0 calor de adsorção, por envolver ligações químicas, é da mesma ordem de grandeza dos calores de reacção (entre 200 e 840 KJ/mol (Foust, 1980)).

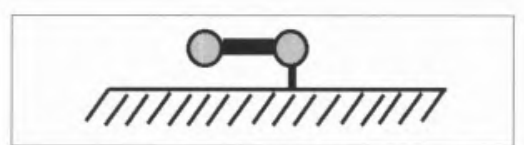

Num processo de adsorção física as forças envolvidas são forças do tipo van der Walls. Não há alteração química das moléculas adsorvidas, podem formar-se camadas moleculares sobrepostas e o calor de adsorção é pequeno (entre $2 \mathrm{e}$ $20 \mathrm{KJ} / \mathrm{mol}$, (Foust, 1980))

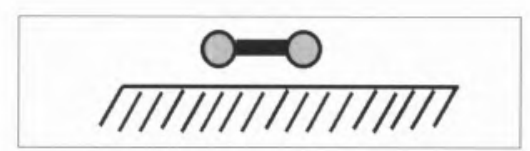

A capacidade de adsorção de um sólido é proporcional à superfície de contacto soluto/sólido. É por este motivo que os adsorventes são usualmente sólidos porosos com elevadas áreas superficiais (p.e. a área específica do carvão activa- do varia entre 800 e $1800 \mathrm{~m}^{2} / \mathrm{g}$ (Le Van et al., 1997).

Para descrever o processo de adsorção recorre-se ao conceito de isotérmica de adsorção. A isotérmica é uma equação que relaciona a quantidade de soluto adsorvido por unidade de massa de sólido (q) com a concentração de soluto em solução (C), numa situação de equilíbrio a temperatura constante. As isotérmicas de Langmuir (equação 1) e de Freundlich (equação 2) são as mais simples e descrevem os resultados experimentais adequadamente para um grande número de sistemas.

Isotérmica de Langmuir

$$
\frac{q}{q_{\max }}=\frac{K C}{1+K C}
$$

Isotérmica de Freundlich

$$
\frac{q}{q_{\max }}=K C^{1 / n}
$$

Nestas equações $q_{\text {máx }}$ representa a quantidade máxima de soluto adsorvido ao sólido e K representa a constante de equilíbrio de adsorção. Conhecendo o valor da constante $\mathrm{K}$ a diferentes temperaturas é possivel determinar a entalpia de adsorção $(\Delta \mathrm{H})$ pela equação de van't Hoff na forma integrada, considerando $\mathrm{DH}$ independente da temperatura.

$$
\ln (K)=-\frac{\Delta H}{R} \frac{1}{T}+c t e
$$




\section{Procedimento experimental}

0 trabalho experimental consiste na determinação das concentrações de equilíbrio de uma solução de Violeta de Cristal (VC) após contacto com areia, a uma temperatura constante, durante um tempo bem definido. Propõe-se que o trabalho seja realizado por vários grupos de alunos, trabalhando cada grupo a uma temperatura diferente, na gama 30 a $60^{\circ} \mathrm{C}$. Desta forma cada grupo determina a constante de equilíbrio a uma temperatura; o tratamento dos resultados de toda a turma permitirá estimar o valor do calor de adsorção.

Para a realização do trabalho é necessário um banho termostático com agitação, um espectrofotómetro ou um colorímetro com um filtro de 590nm e material corrente de laboratório.

A cada grupo é fornecida uma solução aquosa de Violeta de Cristal (aprox. 40 $\mathrm{mg} / \mathrm{L}$ ). Por diluição desta solução cada grupo prepara $200 \mathrm{~mL}$ de 6 a 8 soluções de diferente concentração, na gama 0,4 a $20 \mathrm{mg} / \mathrm{L}$. Estas soluções são usadas para traçar a curva de calibração do espectrofotómetro (Absorvância a 590nm versus Concentração) e para realizar o ensaio de adsorção.
Em 6 (ou 8) erlenmeyers numerados coloca-se $1 \mathrm{~g}$ de areia (rigorosamente pesada) e adicionam-se $100 \mathrm{~mL}$ de uma das soluções diluídas, tal que os diferentes erlenmeyers contenham soluções de diferente concentração. Tampam-se os erlenmeyers e colocam-se no banho termostático à temperatura pretendida, agitando durante 2-3 horas*. Após este período lê-se a absorvância de cada solução, a $590 \mathrm{~nm}$.

\section{Tratamento dos resultados experimentais}

Através da curva de calibração transformar as leituras de Absorvância em concentração $\left(\mathrm{C}_{\mathrm{vc}}, \mathrm{mmol} / \mathrm{m}^{3}\right)$ e calcular a quantidade adsorvida ao sólido (q, $\mathrm{mmol} / \mathrm{Kg}$ ) em cada erlenmeyer, pela equação

$\mathrm{q}=\left(\mathrm{C}_{\mathrm{vCo}}-\mathrm{C}_{\mathrm{vc}}\right) \cdot \mathrm{V} / \mathrm{M}_{\mathrm{ads}}$

onde:

- $\mathrm{C}_{\mathrm{vco}}\left(\mathrm{mmol} / \mathrm{m}^{3}\right)$ representa a concentração inicial de Violeta de Cristal,

- $\mathrm{C}_{\mathrm{vc}}\left(\mathrm{mmol} / \mathrm{m}^{3}\right)$ representa a concentração Violeta de Cristal no equilíbrio,

- $\mathrm{V}\left(\mathrm{m}^{3}\right)$ representa o volume de solução,
- $\mathrm{M}_{\mathrm{ads}}(\mathrm{Kg})$ representa a massa de adsorvente (areia).

Representar a quantidade adsorvida (q) em função da concentração de Violeta de Cristal $\left(\mathrm{C}_{\mathrm{vc}}\right)$, seleccionar a isotérmica mais adequada e determinar os parâmetros característicos, nomeadamente a constante de equilibrio $\mathrm{K}$.

Representar os valores de K (obtidos pelos vários grupos) em função da temperatura absoluta, segundo a equação 3, e estimar a entalpia de adsorção $(\Delta \mathrm{H})$.

\section{Resultados experimentais e discussão}

Na figura 2 apresentam-se resultados típicos obtidos para a adsorção do Violeta de Cristal em areia, a $21^{\circ} \mathrm{C}$. Estes resultados ajustam-se bem à isotérmica de Langmuir e os parâmetros característicos podem ser calculados por regressão linear ou não linear.

\section{Regressão linear}

Os parâmetros $\mathrm{K}$ e q $\mathrm{q}_{\max }$ determinam-se por linearização da isotérmica de Langmuir (equação 1), representando 1/q em função de $1 / C_{v c}$ (equação 5 ) ou $\mathrm{C}_{\mathrm{vc}} / \mathrm{q}$ em função de $\mathrm{C}_{\mathrm{vc}}$ (equação 6 ).

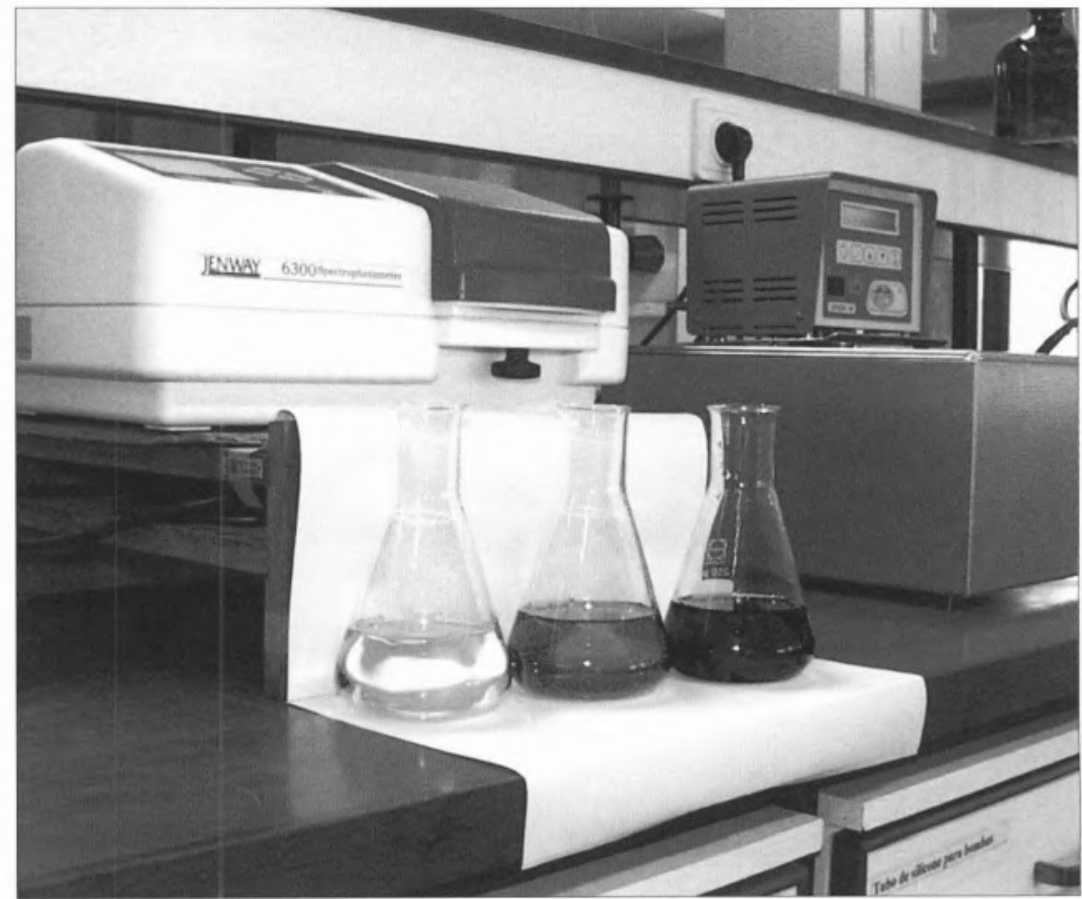



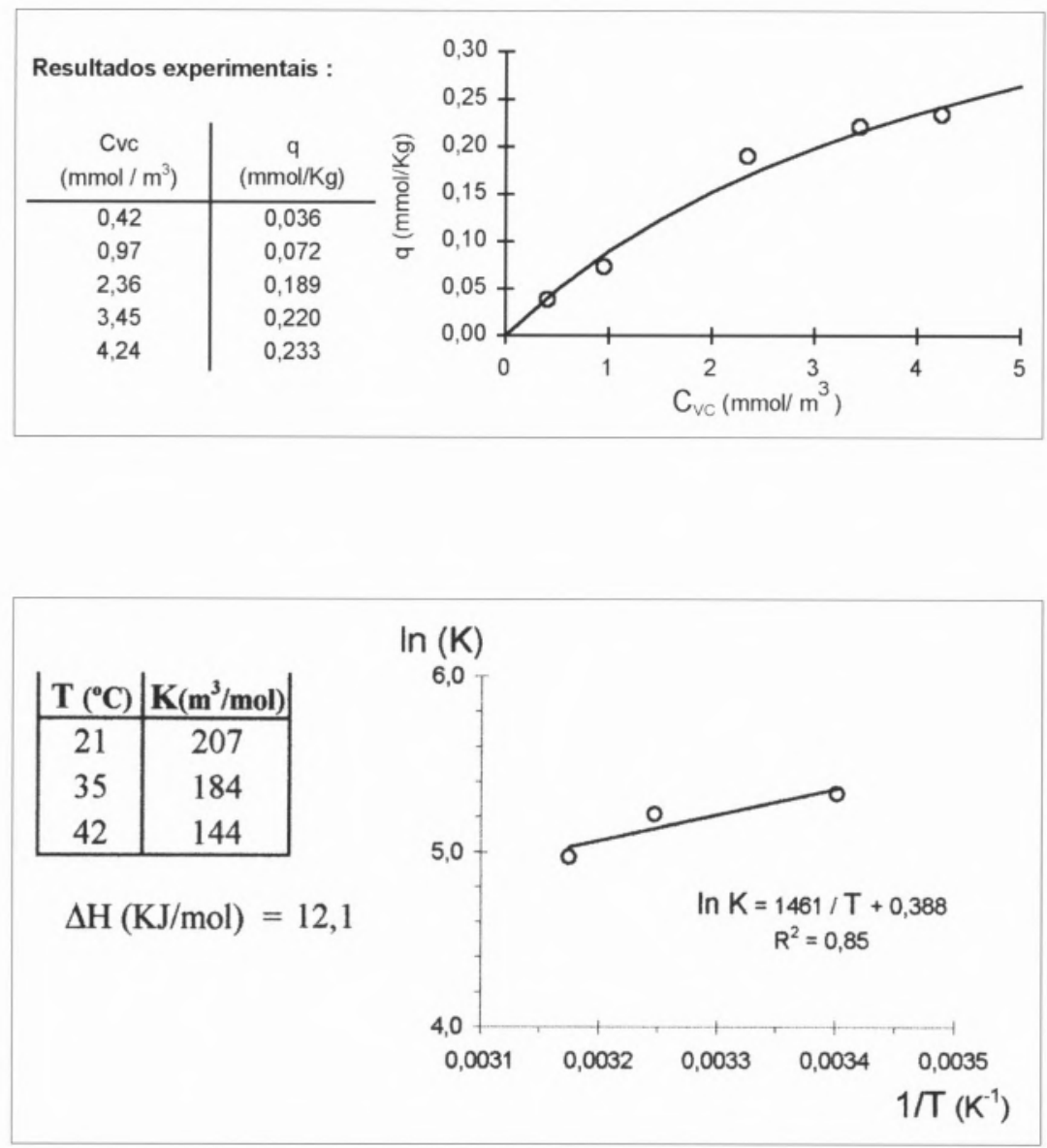

figura 2 Resultados experimentais da adsorção de Violeta de Cristal em areia, a $21^{\circ} \mathrm{C}$, e ajuste da isotérmica de Langmuir obtido por regressão não linear.

$$
\frac{1}{q}=\frac{1}{K q_{\max }} \frac{1}{C \mathrm{vc}}+\frac{1}{q_{\max }}
$$

$$
\frac{C v c}{q}=\frac{1}{q_{\text {máx }}} C \mathrm{vc}+\frac{1}{K q_{\text {máx }}}
$$

Os parâmetros da isotérmica de Langmuir - $\mathrm{K}$ e $\mathrm{q}_{\max }$ - determinados pelas duas equações são muito semelhantes (ver Tabela 1). A equação 6 é recomendada quando os valores de concentração são muito baixos, para evitar a distorção do erro experimental associada ao cálculo $1 / \mathrm{C}_{\mathrm{vc}}$.

\section{Regressão não linear}

A determinação dos parâmetros por regressão não linear pode efectuar-se com

$$
\sum_{i=1}^{n} \Delta q^{2}=\sum_{i=1}^{n}\left(q_{i}^{\text {calc }}-q_{i}^{\exp }\right)^{2}=\sum_{i=1}^{n}\left(q_{m a x} \frac{K \cdot C_{i}^{\exp }}{1+K \cdot C_{i}^{\exp }}-q_{i}^{\exp }\right)^{2}
$$

a ferramenta SOLVER do Excel (Gottfried, 1998), definindo como função objectivo a minimizar o somatório de $\Delta \mathrm{q} 2$, definido pela equação 7. Os parâmetros obtidos para a isotérmica de Langmuir são apresentados na Tabela 1. Verificase que o valor da capacidade máxima de adsorção $q_{\max }$ é semelhante aos valores obtidos por regressão linear mas o valor da constante de equilíbrio é significativamente diferente. Sendo possível recomenda-se a determinação dos parâmetros por regressão não linear.

\section{Determinação do calor de adsorção}

Na figura 3 apresentam-se valores da constante de equilíbrio de adsorção a diferentes temperaturas, obtidos por vários grupos de alunos. Verifica-se que a figura 3-Efeito da temperatura na constante de equilíbrio de adsorçâo de Violeta de Cristal em areia ( $\mathrm{K}$ 's determinados pela isotérmica de Langmuir) e regressão não linear. constante de equilíbrio $\mathrm{K}$ diminui com a temperatura. Esta observação está de acordo com o comportamento exotérmico da adsorção, sendo possível estimar o calor de adsorção pela da equação de van't Hoff na sua forma integrada (equação 3).

O calor de adsorção obtido é muito baixo $(\Delta \mathrm{Hads}=-12.1 \mathrm{KJ} / \mathrm{mol})$ o que permite classificar o processo de adsorção de Violeta de Cristal em areia como adsorção física.

\section{Notas}

* - Sendo a areia um sólido não poroso 2-3 horas é um tempo suficiente para se atingir o equilibrio. Caso não haja disponível um banho com agitação poderá realizar-se uma agitação manual de cada frasco de 15 em 15 minutos, por exemplo. 
Tabela 1

Parâmetros da isotérmica de Langmuir e função objectivo minimizada

Regressão linear

Regressão não linear

Eq. $5 \quad$ Eq. 6

$\begin{array}{lllc}\mathrm{K}\left(\mathrm{m}^{3} / \mathrm{mmol}\right) & 0,13 & 0,14 & 0,21 \\ \mathrm{q}_{\max }(\mathrm{mmol} / \mathrm{Kg}) & 0,68 & 0,68 & 0,52 \\ \Sigma \Delta \mathrm{q} 2 & 9,05 \mathrm{E}-04 & 8,74 \mathrm{E}-04 & 6,96 \mathrm{E}-04\end{array}$

Bibliografia

Foust, A. (1980), Princípios das operações unitárias, (2. ${ }^{a}$ ed.), John Wiley \& Sons, P.37

Le Van, M.D.; Carta, G.; Yon, C.M. (1977), Adsorption and lon-exchange - Section 16 In Perry's Chemical Engineers' Handbook, Editores R H. Perry e D. W. Green, (7. ed.) McGraw-Hill, p.16-9.

Gottfried, B.S. (1998), Spreadsheet Tools for Engineers: Excel 97 version, McGraw-Hill, p.103-150, 177-201.

\section{Técnicas Labosatosiais de Química Videa Cassette}

Com 7 blocos curtos e independentes, este trabalho foi concebido para apoiar as aulas de Técnicas Laboratoriais de Química destinadas a alunos do Ensino Secundário e das cadeiras introdutórias de Química do Ensino Superior. Os procedimentos apresentados são clássicos, simples e adequados para estes níveis de ensino, onde a transparência dos princípios químicos a ilustrar e a necessidade de adopção de boas práticas laboratoriais são da maior importância formativa

\section{Índice}

Pesagem e Preparação de Soluções (11 minutos) Operação de balanças técnicas e de precisão Preparação de soluções rigorosas enão rigorosas

Análise Volumétrica Quantitativa (11 minutos)

Operação com pipetas e buretas

Titulações manuais

Recristalização e Fïltração (24 minutos)

Recristalização por dissolução e arrefecimento

Filtração em papel e à trompa

Filtração a quente

Extraç̧ãoLíquido-Liquido (7 minutos)

Operação com ampolas de decantação

Destilação (23 minutos)

Destilações simples, fraccionada,

a pressão reduzida e por arrastamento de vapor

TLC e Pontos de Fusão (9 minutos)

Cromatografia de Camada Fina

Enchimento de capilares para p.f.

Sopragem de Vidro (11 minutos)

Estirar tubos capilares

Cortar e dobrar tubos de vidro

Demonstração do fabrico e reparação de material de vidro executada por sopradores de vidro profissionais do IST.

$\quad$ Ficha Técnica
Coordenação
Carlos Romão
Hermínio Diogo
Texto e Locução
Carlos Romão
Execução Laboratorial
Hermínio Diogo
João Paulo Telo
Conceição Mesquita
João Ferreira
Carlos Nuno
José Luis Rodrigues
Filmagem e Montagem Video
Luis Raposo
Anabela Martins
Hermínio Costa
Joaquim Pinto
Produção
Núcleo de Audio Visuais
do IST
Apresentação e Duração
lh 36min; Cassette VHS
Distribuição Exclusiva
Sociedade Portuguesa de Quimica

Encomendas à SPQ, Av. da República 37, 4 $4^{\circ}, 1050$ Lisboa

Tel: 217934637 / Fax: 217952349

Preço: Instituições e não sócios $7500 \$ 00$ + IVA + portes

Sócios $6000 \$ 00+$ IVA + portes 